\title{
COMPUTATIONAL INVERSE COHERENT WAVE FIELD IMAGING
}

\author{
Vladimir Katkovnik, Artem Migukin and Jaakko Astola \\ Department of Signal Processing, Tampere University of Technology, \\ Tampere, Finland, e-mail: (firstname.secondname)@tut.fi.
}

\begin{abstract}
We consider reconstruction of a wave field distribution in an input/object plane from data in an output/diffraction (sensor) plane. For the forward propagation the matrix form of the discrete diffraction transform $(\mathcal{D D} \mathcal{T})$ originated in [1] and [2] is used. This "matrix $\mathcal{D D} \mathcal{T}$ " is aliasing free and precise for pixel-wise invariant object and sensor plane distributions. A contribution of this paper concerns a study of the backward wave field propagation as an inverse problem for the diffraction kernel. The analysis of the conditioning of the transfer $\mathcal{D D} \mathcal{T}$ matrices is presented in order to find when the perfect reconstruction of the object wavefield distribution is possible. This condition number can be used as an indicator of the accuracy of the wave field reconstruction. Simulation experiments show that the developed inverse propagation algorithm demonstrates an improved accuracy as compared with the standard convolutional and discrete Fresnel transform algorithms.
\end{abstract}

Index Terms - Digital holography, inverse coherent imaging, wave field propagation and reconstruction.

\section{INTRODUCTION}

A wave field reconstruction from intensity and phase measurements is one of the basic problems in digital coherent imaging and holography (e.g. [3]). In a typical holography scenario one distinguishes input/object and output/image planes (Fig.1). The object plane is a source of light radiation or reflection propagating along the optical axis. The image plane is parallel to the object plane with a distance $z=d$ between the planes. The discrete diffraction transform $(\mathcal{D D} \mathcal{T})$ links discrete pixelated values of the object and sensor distributions. It is the so-called discrete-to-discrete modeling which is aliasing free and accurate for a pixel-wise invariant object distribution and an image from a pixelated sensor [1],[2].

In this paper the reconstruction of the object distribution from a distribution given in the image plane is considered as an inverse problem. Depending on the pixel size, the size of the sensor and the distance between the object and image planes $\mathcal{D D} \mathcal{T}$ matrices can be very ill-conditioned what makes

This work was supported by the Academy of Finland, project No. 213462 (Finnish Centre of Excellence program 2006 - 2011). the reconstruction of the object distribution difficult or even impossible. The $\mathcal{D D} \mathcal{T}$ forward matrix transform modeling is a natural and very productive tool to study limitations of the wave field reconstruction and to develop novel effective algorithms. In this paper we study the accuracy of this inverse reconstruction technique and link it with the conditioning of the transfer matrices of $\mathcal{D D} \mathcal{T}$.

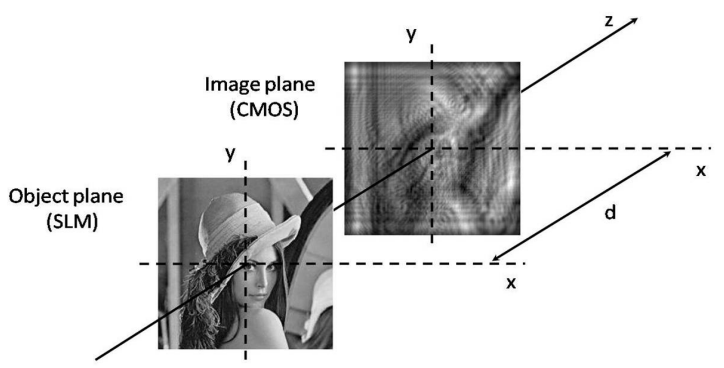

Fig. 1. Principal setup of wave field propagation and reconstruction.

\section{STANDARD MODELING OF WAVE FIELD PROPAGATION}

Let $u_{d}(x, y)$ be a complex-valued $2 D$ wave field defined in a image plane $z=d$ as a function of the lateral coordinates $x$ and $y$. According to the scalar diffraction theory there is the diffraction operator $\mathcal{D}_{z}$ which links this sensor wave field distribution with the object wave field $u_{0}(x, y)$ at $z=0$ as $u_{d}(x, y)=\mathcal{D}_{d}\left\{u_{0}\right\}=\left\{g_{z} \circledast u_{0}\right\}(x, y)$. We assume that $z \gg \sqrt{x^{2}+y^{2}}$ and use the Fresnel approximation of the diffraction kernel (point spread function (PSF)) $g_{z}$ as

$$
g_{z}(x, y)=\frac{\exp (j 2 \pi z / \lambda)}{j \lambda \cdot z} \exp \left[j \frac{\pi}{\lambda z}\left(x^{2}+y^{2}\right)\right],
$$

where $\lambda$ is a wavelength.

The following important points define a specific of the considered coherent wave field convolution and the corresponding inverse problem. First, PSF is complex valued with the absolute value invariant with respect to $x$ and $y$. Thus, this PSF has an infinite support. The blur effects in $u_{d}$ are 
obtained and define by the phase characteristic of PSF (not by the module as it is in the standard deblurring settings). These moments define essential specific features of the considered deconvolution.

The kernel of the inverse diffraction operator (which is known analytically, e.g. [3]) defines the estimate of $u_{0}$ from $u_{d}$ as $\hat{u}_{0}(x, y)=\mathcal{D}_{-d}\left\{u_{d}\right\}$. This estimate is perfect (precise for any $\left.u_{0}\right), \hat{u}_{0}=u_{0}$, provided that $u_{d}(x, y)$ is given for all $(x, y) \in R^{2}$ and non-perfect ( $\hat{u}_{0}$ is not identical to $u_{0}$ ), for a finite size sensor [3]. The standard algorithms (convolutional methods, Fourier and Fresnel transforms, etc.) are based on digital approximations of the inverse operator $\mathcal{D}_{-d}$ and can be given in the form $\hat{u}_{0}(x, y)=\hat{\mathcal{D}}_{-d}\left\{\hat{u}_{d}\right\}$, where the hat stands for the discrete approximation of $\mathcal{D}_{-d}$, where $\hat{u}_{d}$ is a hologram detected on a finite size sensor. All these discrete algorithms inherit limitations following from using for a finite size sensor the operator $\mathcal{D}_{-d}$ derived for the infinite size sensor. Contrary to this approach the inverse imaging paradigm considered in this paper is based on digital approximation of a truncated version of the forward propagation operator $\mathcal{D}_{d}, \hat{u}_{d}(x, y)=\hat{\mathcal{D}}_{d}^{t r}\left\{u_{0}\right\}$. The $\mathcal{D}_{d}^{t r}$ is shift-varying and depending on parameters can be very ill-conditioned contrary to $\mathcal{D}_{d}$, which is shift-invariant and well-conditioned. The inverse imaging paradigm is based on inverse of this operator $\hat{\mathcal{D}}_{d}^{t r}$

\section{MATRIX DISCRETE DIFFRACTION TRANSFORM}

The standard techniques mentioned in the previous section consider discrete models as approximations for forward and backward wave field propagation integrals. In the approach proposed in [1], [2], further developed and studied in this paper, we follow different ideas.

In our model we assume that the input and output signals are pixel-wise constant distributions defined by values in pixels of the digital devices: for instance, an SLM and a sensor respectively. In [2] we presented a novel algebraic matrix model for $\mathcal{D D} \mathcal{T}$ called as Matrix Discrete Diffraction Transform $(\mathcal{M}-\mathcal{D D} \mathcal{T})$, which gives an accurate discreteto-discrete forward propagation modeling for pixel-wise invariant distributions. In contrast to standard methods we are free from restrictions on the size of the pixel and image. The pixels and images in the object and image planes can be of different size and rectangular, but fixed (physical restriction on certain devices with specific parameters).

\subsection{Forward $\mathcal{M}-\mathcal{D} \mathcal{D} \mathcal{T}$ modeling}

Let pixels in object and sensor planes be rectangular of the sizes $\left(\Delta_{y, 0} \times \Delta_{x, 0}\right)$ and $\left(\Delta_{y, z} \times \Delta_{x, z}\right)$, respectively. The sizes of the images in the object $\mathbf{u}_{0}$ and sensor $\mathbf{u}_{z}$ planes measured in pixels can be also different $N_{y, 0} \times N_{x, 0}$ and $N_{y, z} \times N_{x, z}$. According to the formulas (24)-(26) from [2] the forward wave field propagation for the kernel (1) can be presented in the matrix form as

$$
\begin{aligned}
& \mathbf{u}_{z}=\mu \cdot \mathbf{A}_{y} \cdot \mathbf{u}_{0} \cdot \mathbf{A}_{x}^{T}, \mu=\exp (j 2 \pi z / \lambda) /(j \lambda z) \\
& \mathbf{A}_{y}[k, s]=\frac{\mathbf{C}_{y}[k, s]}{\Delta_{y, z}} \int_{-\Delta_{y, z} / 2}^{\Delta_{y, z} / 2} \int_{-\Delta_{y, 0} / 2}^{\Delta_{y, 0} / 2} d \xi d \xi^{\prime} \times \\
& \exp \left(j \frac{2 \pi}{\lambda z}\left(k \Delta_{y, z}-s \Delta_{y, 0}\right)\left(\xi^{\prime}+\xi\right)\right) \cdot \exp \left(j \frac{\pi}{\lambda z}\left(\xi^{\prime}+\xi\right)^{2}\right) \\
& \mathbf{A}_{x}[l, t]=\frac{\mathbf{C}_{x}[l, t]}{\Delta_{x, z}} \int_{-\Delta_{x, z} / 2}^{\Delta_{x, z} / 2} \int_{-\Delta_{x, 0} / 2}^{\Delta_{x, 0} / 2} d \eta d \eta^{\prime} \times \\
& \exp \left(j \frac{2 \pi}{\lambda z}\left(l \Delta_{x, z}-t \Delta_{x, 0}\right)\left(\eta^{\prime}+\eta\right)\right) \cdot \exp \left(j \frac{\pi}{\lambda z}\left(\eta^{\prime}+\eta\right)^{2}\right)
\end{aligned}
$$

where $k=-N_{y, z} / 2, \ldots, N_{y, z} / 2-1, s=-N_{y, 0} / 2, \ldots, N_{y, 0} / 2-$ $1, l=-N_{x, z} / 2, \ldots, N_{x, z} / 2-1, t=-N_{x, 0} / 2, \ldots, N_{x, 0} / 2-1$ and $\mathbf{C}_{y}[k, s]=\exp \left(j \frac{\pi}{\lambda z}\left(k \Delta_{y, z}-s \Delta_{y, 0}\right)^{2}\right), \mathbf{C}_{x}[l, t]=$ $\exp \left(j \frac{\pi}{\lambda z}\left(l \Delta_{x, z}-t \Delta_{x, 0}\right)^{2}\right)$.

The formula (2) defines what we call the matrix discrete diffraction transform $(\mathcal{M}-\mathcal{D D} \mathcal{T})$.

The $\mathbf{A}_{y}[k, s], \mathbf{A}_{x}[l, t]$ becomes shift-invariant depending on the differences of the arguments $k-l$ and $s-t$ as soon as the pixels in the object and sensor planes take equal sizes, $\Delta_{x, z}=\Delta_{x, 0}=\Delta_{x}$ and $\Delta_{y, z}=\Delta_{y, 0}=\Delta_{x}$. Overall, in this case $\mathcal{M}-\mathcal{D} \mathcal{D} \mathcal{T}$ becomes simpler because the matrices $\mathbf{A}_{y}, \mathbf{A}_{x}$ are symmetrical $\mathbf{A}_{y}[k, s]=\mathbf{A}_{y}[k-s]=\mathbf{A}_{y}[s-k]$, $\mathbf{A}_{x}[l, t]=\mathbf{A}_{x}[l-t]=\mathbf{A}_{x}[t-l]$. If $\Delta_{y}=\Delta_{x}$ and $N_{y}=N_{x}$ then $\mathbf{A}_{x}=\mathbf{A}_{y}$.

\subsection{Backward (inverse) modeling and perfect recon- struction}

The inverse of $\mathcal{M}-\mathcal{D D} \mathcal{T}$ is able to give the perfect reconstruction of the pixel-wise object distribution if the forward operator $\mathcal{M}-\mathcal{D D} \mathcal{T}$ is non-singular.

In particular, it is shown in [2] that for rectangular object and sensor planes and non-singular well-posed $\mathbf{A}_{y}$ and $\mathbf{A}_{x}$ the perfect reconstruction is given by the formula

$$
\hat{\mathbf{u}}_{0}=\frac{1}{\mu}\left(\mathbf{A}_{y}^{H} \mathbf{A}_{y}\right)^{-1} \mathbf{A}_{y}^{H} \mathbf{u}_{z} \mathbf{A}_{x}^{*}\left(\mathbf{A}_{x}^{T} \mathbf{A}_{x}^{*}\right)^{-1},
$$

where $\left({ }^{H}\right)$ stands for the Hermitian conjugate, $\mathbf{A}_{y}^{H}=\left(\mathbf{A}_{y}^{*}\right)^{T}$.

However, numerical experiments demonstrate that depending on the distance $z$, the pixels' sizes, and object and sensor sizes the matrices $\mathbf{A}_{y}$ and $\mathbf{A}_{x}$ can be extremely illconditioned. One of the efficient ways to deal with this ill-conditioning is to involve a prior information on the object distribution in question. In [2] we show the efficiency of the inverse using the standard Tikhonov's regularizator [4]. Instead of solution of the equation (2) we are looking for the regularized estimate of $\mathbf{u}_{0}$ defined by minimization of the quadratic criterion $L=\left\|\mathbf{u}_{z}-\mu \mathbf{A}_{y} \mathbf{u}_{0} \mathbf{A}_{x}^{T}\right\|_{F}^{2}+\alpha^{2}\left\|\mathbf{u}_{0}\right\|_{F}^{2}$, 


$$
\hat{\mathbf{u}}_{0}=\arg \min _{\mathbf{u}_{0}} L,
$$

where the quadratic Frobenius matrix norm is defined by the formula $\left\|\mathbf{u}_{0}\right\|_{F}^{2}=\sum_{k, l}\left|u_{0}(k, l)\right|^{2}$.

The regularization penalty $\left\|\mathbf{u}_{0}\right\|_{F}^{2}$ enables a bounded values for reconstructed $\mathbf{u}_{0}$. The regularization parameter $\alpha^{2}$ controls the level of the regularization or the smoothness of $\hat{\mathbf{u}}_{0}$ imposed by this penalization [4].

The regularized inverse $\hat{\mathbf{u}}_{0}$ defined as a minimizer of $L$ approximately could be calculated as [2]

$$
\begin{aligned}
& \hat{\mathbf{u}}_{0}=\mu^{*}\left(|\mu| \mathbf{A}_{y}^{H} \mathbf{A}_{y}+\alpha \mathbf{I}\right)^{-1} \times \\
& \mathbf{A}_{y}^{H} \mathbf{u}_{z} \mathbf{A}_{x}^{*}\left(|\mu| \mathbf{A}_{x}^{T} \mathbf{A}_{x}^{*}+\alpha \mathbf{I}\right)^{-1}, \alpha>0 .
\end{aligned}
$$

Comparing the last equation with (4) we note that the inverse of $\mathbf{A}_{y}^{H} \mathbf{A}_{y}$ and $\mathbf{A}_{x}^{T} \mathbf{A}_{x}^{*}$ is replaced by the inverse of their regularized versions $|\mu| \mathbf{A}_{y}^{H} \mathbf{A}_{y}+\alpha \mathbf{I}$ and $|\mu| \mathbf{A}_{x}^{T} \mathbf{A}_{x}^{*}+\alpha \mathbf{I}$. In general, the regularized inverse (6) gives a biased estimate of the true distribution. Smaller $\alpha$ means a smaller bias. However, too small $\alpha$ is not applicable as the estimate $\hat{\mathbf{u}}_{0}$ can become very noisy and even completely destroyed because of amplification observation noises as well as round off calculation errors, i.e. because of the effects typical for illconditioned problems.

One of the main pragmatical results of this paper is that we show that the regularized inverse imaging is able to give results which are essentially better that it can be achieved on the base of the standard techniques.

It is proved in [2] that the perfect reconstruction $\hat{\mathbf{u}}_{0}=\mathbf{u}_{0}$ can be achieved for any size of the object and image planes and the pixels in these planes if the following sampling conditions are fulfilled:

$$
N_{y}=\frac{\lambda \cdot z}{\Delta_{y, 0} \Delta_{y, z}}, N_{x}=\frac{\lambda \cdot z}{\Delta_{x, 0} \Delta_{x, z}},
$$

In this case transfer matrices $\mathbf{A}_{y}$ and $\mathbf{A}_{x}$ are well-posed and the regularization is not required $(\alpha \rightarrow 0)$.

\section{SIMULATION EXPERIMENTS FOR WAVE FIELD RECONSTRUCTION}

It is assumed that the images in object and sensor planes are square but can be of different size, $N_{y, 0}=N_{x, 0}=N_{0}=256$ and $N_{y, z}=N_{x, z}=N_{z}=q N_{0}, q \geq 1$. Thus, the image in the object plane is always smaller than or equal to that in the object plane with the ratio of the sizes denoted as $q=N_{z} / N_{0}$. It follows from general speculations that the better accuracy of reconstruction for the object plane can be obtained for the larger sensor image (larger $q$ ). While this statement is quite obvious, one of the problems addressed in simulation is the influence of the size parameter $q$ (e.g. $q=\{1,2,4\}$ ) on the condition numbers of the $\mathcal{D D} \mathcal{T}$ matrices and on the accuracy of the image reconstruction. It is assumed that all pixel are square of sizes $\Delta_{y, 0}=\Delta_{x, 0}=\Delta_{0}$ and $\Delta_{y, z}=\Delta_{x, z}=\Delta_{z}$ equal to $7.4 \mu \mathrm{m}$.

The condition number of the square complex valued matrices $\mathbf{A}_{y}^{H} \mathbf{A}_{y}\left(N_{y, 0} \times N_{y, 0}\right)$ or $\mathbf{A}_{x}^{T} \mathbf{A}_{x}^{*}\left(N_{x, 0} \times N_{x, 0}\right)$ are calculated as a relation of the maximum singular value of this matrices to the minimum one, cond $=s_{\max } / s_{\min }$.

We introduce the so-called "in-focus" distance calculated according to the sampling condition (7) as $\left.d_{f}\right|_{q}=N_{z} \cdot \Delta_{z}$. $\Delta_{0} / \lambda$ [2]. In order to emphasize the dependence of the "infocus" distance on the parameter $q$ we will use notation $\left.d_{f}\right|_{q}$ where the values of $q$ is shown. It has been found that for this distance the condition numbers (cond) achieve minimum values, and the formula (4) enables a high-accuracy reconstruction without regularization. With these parameters and for square images $\mathbf{A}_{y}=\mathbf{A}_{x}$, and the matrices $\mathbf{A}_{y}^{H} \mathbf{A}_{y}$ and $\mathbf{A}_{x}^{T} \mathbf{A}_{x}^{*}$ have equal condition numbers. In our experiments we assume the amplitude modulation of the object distribution, defined by the lena test image.

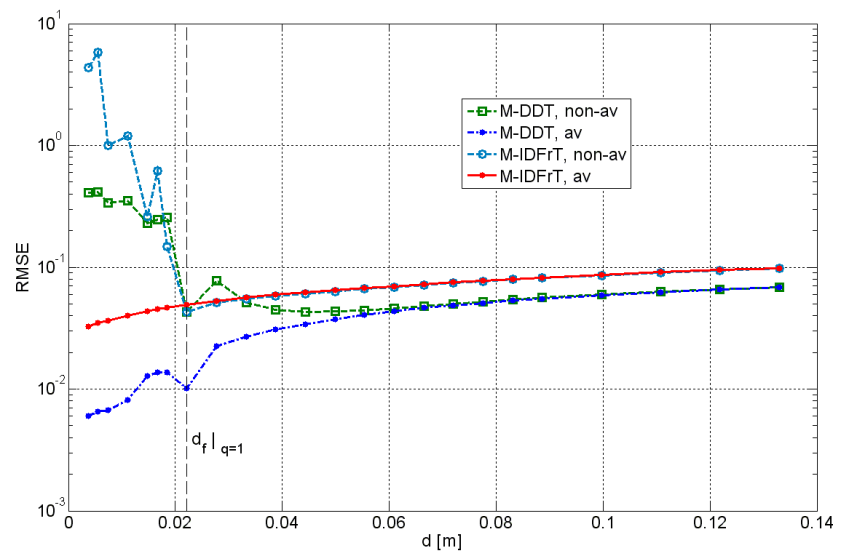

Fig. 2. Accuracy of the wave field reconstruction via RMSE versus distance for $M-D D T$ and $M-I D F r T$ methods with averaged (av) and nonaveraged (non-av) matrices.

Fig. 2 illustrates the accuracy (characterized by $R M S E$ values) obtained by the considered inverse imaging technique versus the best standard one known as the inverse discrete Fresnel transform (IDFrT). To study the accuracy of the latter algorithm for the varying distance $z=d$ provided that the pixel sizes are fixed we use here the matrix version of $M-I D F r T$ (see [2] for details).

In order to separate the effects of integration in (3) and of regularized inverse we consider estimation with the "averaged" matrices $\mathbf{A}_{y}$ and $\mathbf{A}_{x}$ as they are defined in (3) and their "nonaveraged" versions corresponding to small $\Delta_{0}$ and $\Delta_{z}$ when integration in the formulas (3) is dropped. First, note that the averaging is essential and indeed improves the accuracy for $d<\left.d_{f}\right|_{q}$ only, and it is true for the both types of the algorithms the regularized inverse and $M-I D F r T$. The accuracy effects of the inverse is obvious for all distances 
with improvement in RMSE more than $40 \%$.

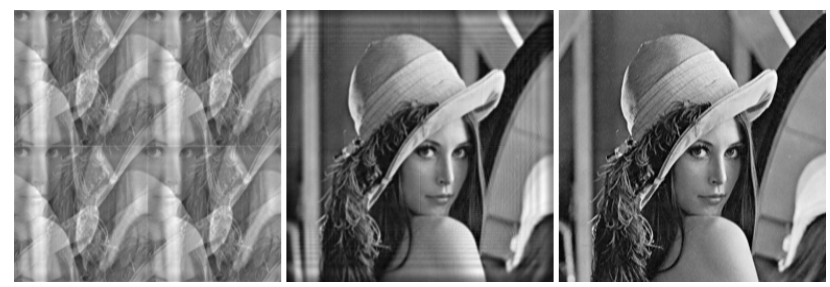

Fig. 3. Object wave field reconstruction (amplitude distribution) the distance $z=0.5 d_{f}$ : (left) standard inverse Fresnel transform fails with a pattern of clear aliasing effects, (center) $M-I D F r T$ with averaged matrices and (right) inverse $M-D D T$ give a good quality aliasing free reconstruction.

The Fig. 3 demonstrates the ability to obtain a highquality imaging in cases, when the standard techniques fail due to the aliasing effects. The middle image shows the results obtained by $M-I D F r T$ using the averaged matrices. This modification of the standard techniques essentially improves the result obtained with no averaging (left image). However, gives the imaging which is worse than obtained using the inverse imaging techniques and averages matrices (right image).

In our next results we demonstrate that the condition number of the matrix $\mathbf{A}_{y}^{H} \mathbf{A}_{y}$ (shown in Fig. 5) can be used for prediction of the accuracy (with RMSE shown in Fig. 4). All these curves are given versus the distance $z=d$. The $R M S E$ accuracy takes minimum value when the condition number is small. These results are shown for various sensor redundancy parameter $q$. Larger $q$ results in a larger area for $d$ where the conditioning is small and respectively $R M S E$ is minimal.

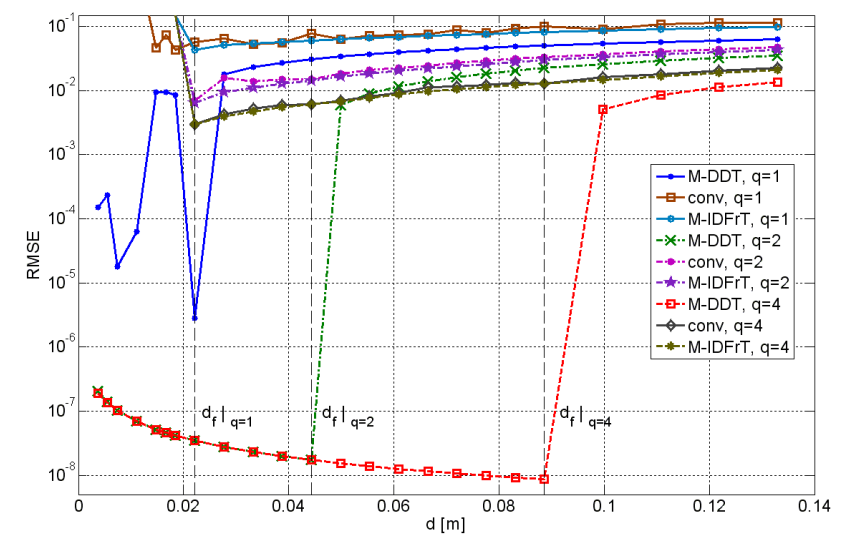

Fig. 4. RMSE accuracy for different methods versus the distance $d, q=1,2,4$.

These experiments are produced for different algorithms and the advantage of the regularized inverse $\mathcal{M}-\mathcal{D D} \mathcal{T}$ algorithm (6) for all distances and corresponding $q$ over the con- ventional algorithms is obvious.

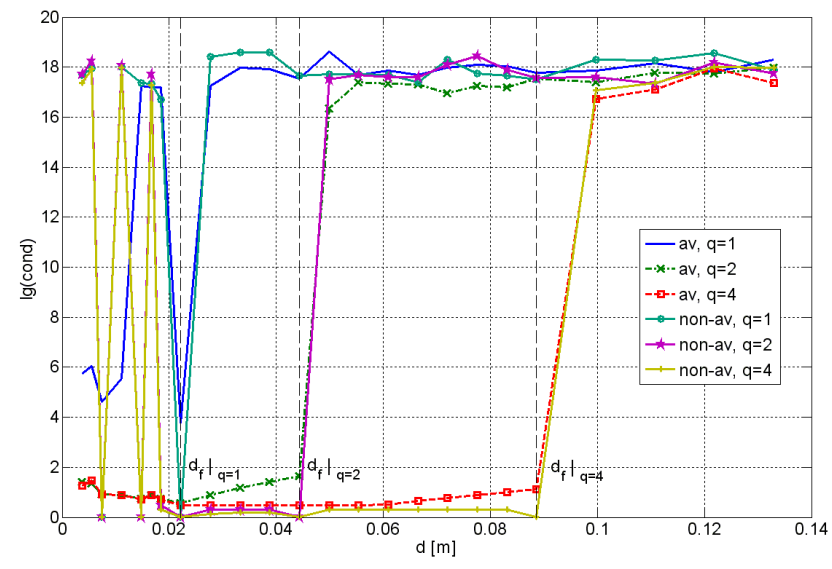

Fig. 5. Condition numbers cond (in log scale) versus the distance $d$ for averaged and non-averaged matrices, $q=1,2,4$.

The condition number is small for all sensor sizes, if $d<\left.d_{f}\right|_{q}$. For these "smaller" distances we obtain a high-accuracy (nearly perfect) reconstruction. As soon as the condition number grows rapidly for $d>\left.d_{f}\right|_{q=2}$ and $d>\left.d_{f}\right|_{q=4}$, the accuracy of reconstruction is correspondingly going down. However it is clear that a larger sensor size $(q>1)$ results in a better accuracy for all methods of the wave field reconstruction. A similarity in behavior of the curves in Fig. 4 and Fig.5 confirms that a study of the condition number gives a clear indication of the accuracy of reconstruction and can be used for optimization of optical setups and sensors.

Overall the above results demonstrate the advantage of the proposed inverse imaging technique for application in holography based problems dealing with the coherent wave field reconstruction. It shown that the condition number can be used for design and optimization of optical setups and in image processing techniques.

\section{REFERENCES}

[1] V. Katkovnik, J. Astola, and K. Egiazarian , "Discrete diffraction transform for propagation, reconstruction, and design of wavefield distributions," Appl. Opt, vol. 47, no. 19, pp. 3481-3493 (2008).

[2] V. Katkovnik, A. Migukin, and J. Astola, "Backward discrete wave field propagation modeling as an inverse problem: toward perfect reconstruction of wave field distributions," Appl. Opt. vol. 48, no. 18, pp. 3407-3423 (2009).

[3] J. W. Goodman, Introduction to Fourier Optics (McGraw-Hill, Inc, New York, Second Edition, 1996).

[4] Tikhonov A.N. and V.Y. Arsenin. Solution of ill-posed problems (Wiley, New York, 1977). 